\title{
Improved survival with double platinum therapy transcatheter arterial infusion using cisplatin and transcatheter arterial chemoembolization using miriplatin for BCLC-B hepatocellular carcinoma
}

\author{
TORU ISHIKAWA, SATOSHI ABE, TAKAYUKI WATANABE, YUJIRO NOZAWA, \\ TOMOE SANO, AKITO IWANAGA, KEIICHI SEKI, TERASU HONMA and TOSHIAKI YOSHIDA \\ Department of Gastroenterology and Hepatology, Saiseikai Niigata Daini Hospital, Niigata 950-1104, Japan
}

Received January 26, 2016; Accepted August 4, 2016

DOI: $10.3892 / \mathrm{mco} .2016 .998$

\begin{abstract}
Transcatheter arterial chemoembolization (TACE) is the standard therapy for Barcelona Clinic Liver Cancer (BCLC) classification intermediate stage B hepatocellular carcinoma (HCC). However, other transcatheter methods, such as transcatheter arterial infusion (TAI), also play an important role in the treatment of advanced HCC. Although it has been reported that TAI with a high concentration of a fine-powder formulation of cisplatin (DDP-H) reduced intrahepatic recurrence and improved survival, the combined use of TAI with DDP-H and TACE has not yet been investigated. The aim of this study was to evaluate which TACE chemotherapeutic agent in combination with DDP-H TAI contributes more to improved survival in BCLC-B HCC. Survival was analyzed in 55 patients who underwent DDP-H TAI and TACE for BCLC-B HCC. The patients were classified into two groups; Epirubicin was used as the TACE agent in 29 patients, whereas miriplatin was used in 26 patients. The cumulative survival rates at 1 and 2 years were 66.4 and $36.0 \%$ in the epirubicin and 95.8 and $61.30 \%$ in the miriplatin group, respectively. Survival time was significantly prolonged in the miriplatin group compared with that in the epirubicin group. Multivariate analysis identified Child-Pugh classification and up-to-seven criteria as factors affecting survival. In addition, the selection of miriplatin as the TACE chemoagent was the treatment factor that most significantly affected survival. Thus, double-platinum therapy with DDP-H TAI and miriplatin TACE may be a useful treatment strategy for improving survival in BCLC-B HCC patients.
\end{abstract}

Correspondence to: Dr Toru Ishikawa, Department of Gastroenterology and Hepatology, Saiseikai Niigata Daini Hospital, 280-7 Teraji, Niigata 950-1104, Japan

E-mail: toruishi@ngt.saiseikai.or.jp

Key words: Barcelona Clinic Liver Cancer classification, transcatheter arterial chemoembolization, hepatocellular carcinoma, chemoagents, platinum

\section{Introduction}

Transcatheter arterial chemoembolization (TACE) is currently the standard first-line treatment for Barcelona Clinic Liver Cancer (BCLC) stage B hepatocellular carcinoma (HCC) patients $(1,2)$.

Chemotherapeutic agents such as doxorubicin (3), epirubicin (4-6), mitomycin C (7) and cisplatin (8) are combined in TACE for the treatment of HCC; however, their treatment efficacy remains unclear.

We previously reported that whole-liver transcatheter arterial infusion (TAI) with a high concentration of a fine-powder formulation of cisplatin (DDP-H) (IA-call ${ }^{\circledR}$; Nippon Kayaku Co. Ltd., Tokyo, Japan) prior to radical local treatment inhibited intrahepatic recurrence of HCC (9), and that whole-liver TAI of DDP-H administered to HCC patients with a Japan Integrated Staging (JIS) score of 0-1 improved survival (10).

The aim of the present study was to evaluate whether epirubicin (Farmorubicin; Pfizer Japan, Tokyo, Japan) or miriplatin (Miripla; Dainippon Sumitomo Pharma, Osaka, Japan) best contributes to survival when used as the chemoagent in TACE in combination with DDP-H whole-liver TAI in patients with primary BCLC-B HCC.

\section{Patients and methods}

Patients. This was a single-center, explorative, cohort study of 55 patients with BCLC-B stage primary treatment-naïve HCC diagnosed between July, 2004 and March, 2015. The survival time and predictive factors were analyzed in 55 BCLC-B stage HCC patients who underwent DDP-H whole-liver TAI in combination with TACE at the Saiseikai Niigata Daini Hospital. Epirubicin (Farmorubicin; Pfizer Japan, Tokyo, Japan) was used as the TACE chemoagent in 29 patients, whereas miriplatin (Miripla; Dainippon Sumitomo Pharma, Osaka, Japan) was used in 26 patients. Epirubicin was selected as first-line treatment before miriplatin approval (October, 2009), and miriplatin was selected as first-line treatment thereafter.

HCC was diagnosed by dynamic contrast-enhanced computed tomography or dynamic contrast-enhanced 
magnetic resonance imaging. The inclusion criteria were as follows: BCLC-B, no prior treatment for $\mathrm{HCC}$, poor candidate for surgery, Eastern Cooperative Oncology Group performance status 0-2, normal electrocardiographic findings, normal serum creatinine level and age $20-85$ years.

Ethics statement. This study was approved by the Institutional Review Board of Saiseikai Niigata Daini Hospital and was conducted in accordance with the principles of the Declaration of Helsinki. All the patients provided written informed consent.

Treatment. In all the patients, the femoral artery was punctured using the Seldinger technique and a 5-F introducer was inserted, followed by insertion of a 5-F catheter. Subsequently, a 3-F microcatheter was advanced into the proper hepatic artery, and DDP-H (IA-call ${ }^{\circledR}$, Nippon Kayaku, Co., Ltd.) 65 mg/m² was infused through the catheter into the whole liver over $30 \mathrm{~min}$. The DDP-H solution was prepared by dissolving DDP-H (100 mg/vial) in $70 \mathrm{ml}$ physiological saline warmed to $50^{\circ} \mathrm{C}$ (cisplatin concentration: $1.43 \mathrm{mg} / \mathrm{ml}$ ). A 5-hydroxytryptamine-3 receptor antagonist and corticosteroids were administered during DDP-H infusion to reduce emesis. To prevent renal toxicity, hydration was performed by intravenous drip infusion of fluids (500 $\mathrm{ml}$ prior to DDP-H infusion and $1,500 \mathrm{ml}$ following DDP-H infusion), and the patients were instructed to ingest $\geq 2,000 \mathrm{ml} /$ day of water beginning the day after treatment.

Following DDP-H infusion, superselective TACE was administered to embolize subsectional and/or peripheral nutrient vessels.

The patients were divided into two groups according to the anticancer agent used in TACE: A total of 29 patients were included in the epirubicin group and 26 patients in the miriplatin group.

When TACE was performed with epirubicin, one 50-mg vial of epirubicin was dissolved in 1-2 $\mathrm{ml}$ of non-ionic contrast agent, and a suspension was prepared by pumping a maximum of $50 \mathrm{mg}$ of epirubicin and 2-8 $\mathrm{ml}$ of lipiodol between 2 syringes 10-20 times. When TACE was performed using miriplatin, one $70-\mathrm{mg}$ vial of miriplatin was dissolved in $4 \mathrm{ml}$ of lipiodol, and a suspension was prepared containing a maximum of $140 \mathrm{mg}$ of miriplatin and $8 \mathrm{ml}$ of lipiodol. In both groups, the amount of suspension used was determined according to the size of the nodule [the suspension was administered until it filled the tumor blood vessels to a maximum of $6 \mathrm{ml}$ (120 mg miriplatin)]. In all procedures, TACE was performed until stasis of the blood flow in the target artery was observed. Following TACE, angiograms were taken from multiple perspectives to confirm that there was no tumor staining or accumulation of lipiodol in the tumor.

Statistical analysis. The primary endpoint of this study was overall survival. Background clinical characteristics were compared between the epirubicin and miriplatin groups by the Wilcoxon rank sum test or Fisher's exact test. Overall survival was calculated from the date of therapy initiation to the date of death, and survival curves were constructed using the Kaplan-Meier method. Overall survival was compared between the two groups by the log-rank test and generalized Wilcoxon test. Prognostic factors were identified by univariate and multivariate analyses using the Cox proportional hazards model. Values of $\mathrm{P}<0.05$ were considered to indicate statistically significant differences. Furtheremore, the time to progression (TTP) was calculated from the date of therapy initiation to the date of radiological progression. All analyses were performed using Statistical Analysis System software, version 9.2 (SAS Institute Inc, Cary, NC, USA). Adverse events were evaluated according to the National Cancer Institute Common Terminology Criteria, version 4.0.

\section{Results}

Patient characteristics. There were no significant differences in background clinical characteristics between the epirubicin and miriplatin groups (Table I). The median follow-up period for all 55 patients was 14.2 months (range, 2.2-60.5 months).

Overall survival and TTP. The 1-, 3- and 5-year cumulative survival rates of BCLC-B patients were 80.0, 40.3 and $23.9 \%$, respectively. Significant differences were found in the cumulative 1- and 2-year survival rates, which were 66.4 and $36.0 \%$, respectively, in the epirubicin group vs. 95.8 and $61.3 \%$, respectively, in the miriplatin group (log-rank test, $\mathrm{P}=0.020$; generalized Wilcoxon test, $\mathrm{P}=0.013$; Fig. 1). In the Child $\mathrm{A}$ group, significant differences were observed with respect to cumulative 1- and 2-year survival rates, which were 79.0 and $44.4 \%$, respectively, in the epirubicin group vs. 100.0 and $62.5 \%$, respectively, in the miriplatin group (log-rank test, $\mathrm{P}=0.048$; generalized Wilcoxon test, $\mathrm{P}=0.039$; Fig. 2). Even in patients with up- to-seven criteria deviations, significant differences were detected in the cumulative 1- and 2-year survival rates, which were 56.5 and $25.4 \%$, respectively, in the epirubicin group vs. 93.3 and $40.8 \%$, respectively, in the miriplatin group.

The median TTP remained significantly longer in the miriplatin group compared with that in the epirubicin group (9.4 vs. 4.4 months, respectively).

Univariate and multivariate analyses for factors predicting survival. Univariate analyses demonstrated that Child-Pugh classification, up-to-seven criteria and the chemoagent were significantly associated with survival (Table II). Multivariate analysis identified Child-Pugh classification [B vs. A: hazard ratio $(\mathrm{HR})=0.177,95 \%$ confidence interval $(\mathrm{CI})$ : 0.059-0.534], up-to-seven criteria (beyond vs. within: $\mathrm{HR}=0.104,95 \% \mathrm{CI}$ : $0.018-0.593$ ) and the use of miriplatin vs. epirubicin as the chemoagent $(\mathrm{HR}=4.103,95 \% \mathrm{CI}: 1.267-13.289)$ as independent predictors of survival (Table III). Therefore, miriplatin as the chemoagent was considered to be the most important treatment factor affecting survival.

Adverse events. Treatment-related adverse events were assessed according to the National Cancer Institute Common Terminology Criteria, version 4.0. Adverse events were evaluated as the maximum change in the grade within 3 months after therapy. Grade 3 or 4 adverse events occurred in both the epirubicin and miriplatin groups (Table IV). In the epirubicin group, these events included an elevated aspartate aminotransferase (AST) level in 13 patients $(44.8 \%$ ), elevated alanine aminotransferase (ALT) level in 13 (44.8\%), thrombocytopenia in $4(13.7 \%)$ and hyperbilirubinemia in 1 patient $(3.4 \%)$. In the miriplatin group, these events 


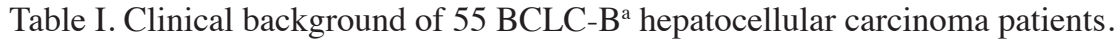

\begin{tabular}{lccc}
\hline Demographic variables & Epirubicin $(\mathrm{n}=29)$ & Miriplatin $(\mathrm{n}=26)$ & P-value \\
\hline Gender, male:female & $24: 5$ & $22: 4$ & 0.85 \\
Age, years & $69.10 \pm 8.09$ & $67.31 \pm 8.97$ & 0.44 \\
Etiology, HBV/HCV/non-B, non-C & $5 / 15 / 9$ & $5 / 12 / 9$ & 0.91 \\
Total bilirubin, mg/dl & $0.87 \pm 0.34$ & $0.77 \pm 0.42$ & 0.29 \\
Serum albumin, g/dl & $3.53 \pm 0.37$ & $3.64 \pm 0.54$ & 0.42 \\
Prothrombin activity, \% & $84.24 \pm 7.262$ & $90.89 \pm 14.99$ & 0.12 \\
ALT, IU/l & $54.56 \pm 37.47$ & $58.38 \pm 42.30$ & 0.72 \\
AST, IU/l & $74.03 \pm 42.94$ & $76.85 \pm 103.98$ & 0.89 \\
Platelet count, x10 $/ \mathrm{mm}^{3}$ & $13.79 \pm 7.25$ & $21.62 \pm 8.53$ & 0.16 \\
AFP, ng/ml & $934.95 \pm 3,236.65$ & $1,604.06 \pm 3,775.83$ & 0.48 \\
DCP, $\mathrm{mAU} / \mathrm{ml}$ & $5,104.88 \pm 12,687.17$ & $7,466.65 \pm 15,111.95$ & 0.53 \\
Child-Pugh score & $6.41 \pm 1.11$ & $5.97 \pm 0.72$ & 0.09
\end{tabular}

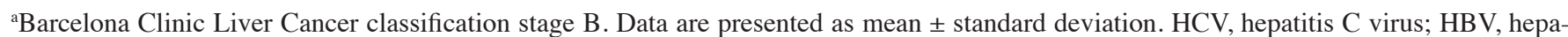
titis B virus; AST, aspartate aminotransferase; ALT, alanine aminotransferase; AFP, $\alpha$-fetoprotein; DCP, des- $\gamma$-carboxy prothrombin.

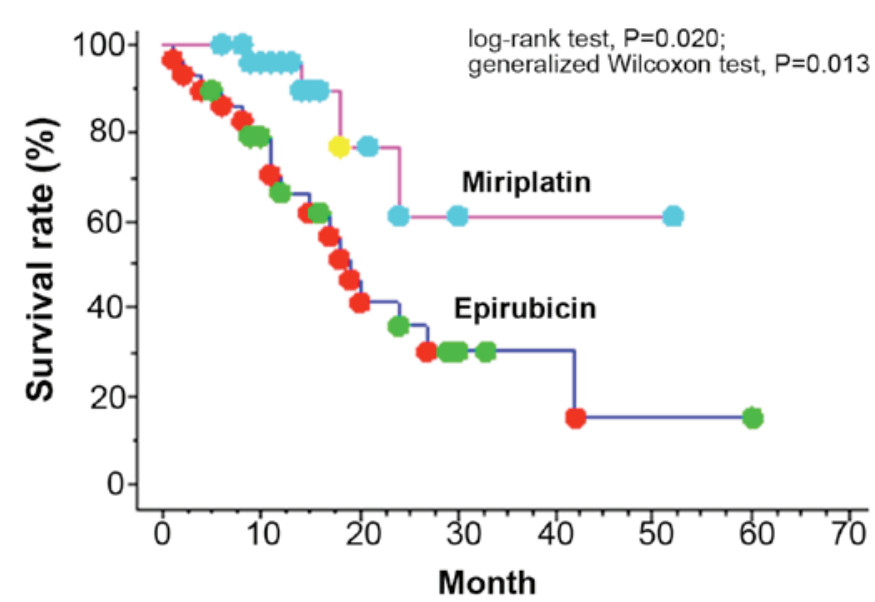

Figure 1. Comparison of overall survival between Barcelona Clinic Liver Cancer (BCLC) stage B hepatocellular carcinoma patients who received transcatherter arterial chemoembolization (TACE) using miriplatin and transcatheter arterial infusion (TAI) with fine-powder formulation of cisplatin (DDP-H), and those who received TACE using epirubicin and TAI with DDP-H. There was a significant difference between the two groups (log-rank test, $\mathrm{P}=0.020$; generalized Wilcoxon test, $\mathrm{P}=0.013$ ).

included an elevated AST level in 5 patients (19.2\%), elevated ALT level in 7 (26.9\%) and thrombocytopenia in 1 patient (3.8\%). The grade 3 or 4 elevation in AST and ALT level was transient in all patients and there was no significant difference in the time to return of the AST and ALT level to normal between the two groups.

Regardless of the use of platinum, there was no grade 3 or 4 renal dysfunction in either group.

\section{Discussion}

The Barcelona Clinic Liver Cancer (BCLC) staging system serves to estimate life expectancy and link the assessment of disease extent and patient status with optimal treatment.

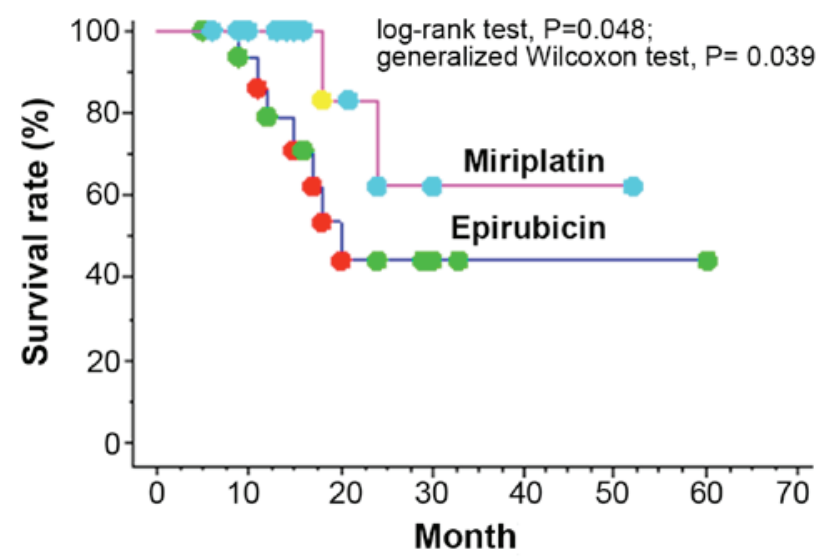

Figure 2. Comparison of overall survival between Barcelona Clinic Liver Cancer (BCLC) stage B hepatocellular carcinoma patients with Child Pugh class A disease, who received transcatherter arterial chemoembolization (TACE) using miriplatin and transcatheter arterial infusion (TAI) with fine-powder formulation of cisplatin (DDP-H), and those who received TACE using epirubicin and TAI with DDP-H. There was a significant difference between the two groups (log-rank test, $\mathrm{P}=0.048$; generalized Wilcoxon test, $\mathrm{P}=0.039$ ).

TACE has been established for the treatment of HCC when surgical resection or other local treatment is not indicated $(11,12)$.

TACE is recommended as the standard treatment for BCLC-B stage HCC.

The major local effects of TACE include local retention of anticancer drugs and lipiodol and the ischemic effects of the embolic material. Various anticancer drugs may be selected, including doxorubicin (3), epirubicin (4-6), mitomycin C (7) and cisplatin (8).

However, there are institutional differences regarding the use of these agents, and the superiority of a specific drug with regard to efficacy has yet to be established. When we investigated the effect of the addition of TAI with DDP-H and carboplatin as pretreatment prior to TACE/radiofrequency 
Table II. Univariate analyses for survival.

\begin{tabular}{lccc}
\hline & & & P-value \\
\cline { 3 - 4 } Variables & & & Generalized \\
Wilcoxon test & Log-rank test & 0.766 \\
\hline Gender & Male/female & 0.902 & 0.354 \\
Age, years & $\geq 70 /<70$ & 0.169 & 0.014 \\
Child-Pugh class & $\mathrm{A} / \mathrm{B}$ & 0.030 & 0.224 \\
AFP, ng/ml & $\geq 400 /<400$ & 0.373 & 0.768 \\
DCP, mAU/ml & $\geq 400 /<400$ & 0.893 & 0.076 \\
Up-to-seven criteria & Beyond/within & 0.018 & 0.309 \\
Albumin, g/dl & $\geq 3.5 /<3.5$ & 0.217 & 0.257 \\
Total bilirubin, $\mathrm{mg} / \mathrm{dl}$ & $<1.0 / \geq 1.0$ & 0.140 & 0.671 \\
Platelet count, $\mathrm{x} 10^{4} / \mathrm{mm}^{3}$ & $\geq 10 /<10$ & 0.417 & 0.013 \\
Chemoagents & Epirubicin $/$ miriplatin & 0.020 & \\
\hline
\end{tabular}

AFP, $\alpha$-fetoprotein; DCP, des- $\gamma$-carboxy prothrombin.

Table III. Multivariate analyses for survival.

\begin{tabular}{lcccc}
\hline Variables & Categories & HR & 95\% CI & P-value \\
\hline Child-Pugh class & B & 0.177 & $0.059-0.534$ & \\
& A & 1 & 0.002 \\
Up-to-seven criteria & Beyond & 0.104 & $0.018-0.593$ & 0.011 \\
Chemoagents & Within & 1 & $1.267-13.289$ \\
\hline
\end{tabular}

HR, hazard ratio; 95\% CI, 95\% confidence interval.

ablation therapy as radical local treatment for stage I/II HCC, we reported that whole-liver TAI using DDP-H significantly inhibited intrahepatic recurrence (9). In addition, in patients with JIS 0-1 stage HCC, survival was significantly prolonged in the group that received whole-liver TAI with DDP-H compared with the group that did not undergo infusion, and the results of the multivariate analyses demonstrated that performing whole-liver TAI with DDP-H was a factor contributing to improved survival (10). Kim et al conducted a retrospective study on the efficacy of arterial cisplatin infusion following TACE in advanced HCC with hepatic vein invasion, and reported that, in the group of patients who underwent arterial cisplatin infusion, survival was significantly prolonged without increased adverse events, compared with that in patients who did not undergo infusion (13). Subgroup analysis demonstrated that cisplatin significantly prolonged survival in patients without extrahepatic metastasis and in patients with a diffuse type of tumor. Based on these findings, arterial infusion of a platinum agent, such as DDP-H, is considered to play an important role as an add-on therapy to TACE in HCC. In the BCLC Guideline, TACE is recommended for BCLC intermediate stage B HCC $(1,2)$.

However, there have been no studies on the efficacy of the chemoagents used when TACE is performed in addition to DDP-H arterial infusion to date. In the present study, we evaluated which chemoagent would be more useful in TACE when used in addition to DDP-H whole-liver TAI.

Epirubicin has been the gold standard among TACE agents. The mechanism of action of epirubicin, similar to that of doxorubicin, is considered to involve DNA intercalation (14). Specifically, as a result of the insertion of epirubicin molecules between bases of DNA, the high-order structure of the DNA is modified, which suppresses DNA polymerase, DNA ligase, topoiomerase I and II, DNA helicase and RNA polymerase activity, and inhibits DNA replication and RNA synthesis. A response rate of $15 \%$ has been reported with the use of hepatic arterial infusion therapy using epirubicin for unresectable HCC (15). However, when administered as an emulsion with lipiodol, a response rate of $42 \%$ has been reported, indicating that epirubicin is more useful when used in combination with lipiodol, rather than as a simple arterial infusion (16).

Miriplatin (cis-[((1R,2R)-1,2-cyclohexanediamine-N, $\mathrm{N}^{\prime}$ )bis(myristato)]-platinum(II)monohydrate; Dainippon Sumitomo Pharma Co., Ltd., Osaka, Japan) is a novel lipophilic cisplatin derivative that may be suspended in lipiodol (17-20). Miriplatin has been developed as a new drug for use in transcatheter arterial chemotherapy for HCC (21). 
Compared with conventionally used water-soluble anticancer agents, miriplatin has a high affinity for lipiodol, which is considered to lead to prolonged retention in the tumor and an increased sustained release effect. Due to its lipophilic nature, miriplatin has a high degree of affinity for lipiodol, and when suspended in lipiodol and administered by hepatic arterial infusion, it is characterized by retention at the tumor site where the platinum component is released.

The therapeutic efficacy of TACE is based on the sustained release of the anticancer agent in the tumor and an ischemic effect due to an embolic agent. Miriplatin has a high capacity for retention at the tumor site due to its high affinity for lipiodol and the fact that only a very small amount is lost in the general circulation. Furthermore, as only a small amount enters the general circulation, renal toxicity is expected to be low and the effectiveness of miriplatin is expected to be high.

However, Miyayama et al reported that superselective TACE using miriplatin resulted in frequent local recurrence, despite less arterial damage compared with TACE using epirubicin and mitomycin C (22). It has also been reported that the local control rate is higher with epirubicin compared with miriplatin (22). However, the short-term effects of miriplatin and epirubicin are reported to be comparable (23). In the present study of BCLC-B HCC, in terms of tumor factors, it was clear that up-to-seven criteria and hepatic reserve play a role in survival; in terms of tumor treatment factors, the combined use of DDP-H and miriplatin contributed to improved survival. We hypothesized that a synergistic antitumor effect of these platinum-based agents has been elicited by combining the sustained release nature of miriplatin and the concentration-dependent property of cisplatin. In a phase I clinical study in which TACE with miriplatin was used in combination with hepatic arterial infusion chemotherapy using cisplatin, investigations were conducted at a dose of $65 \mathrm{mg} / \mathrm{m}^{2}$ of cisplatin in combination with $\leq 120 \mathrm{mg} /$ body of miriplatin, the maximum dose approved in Japan, and it was reported that, in terms of safety, this dose was tolerable (24). In the present study, there were no grade $\geq 4$ adverse events, and no clinically important adverse events; given that there have been no reports of adverse events when miriplatin or cisplatin were used in combination with an embolizing agent, the adverse events in the present study were within the expected range.

The limitation of this study was the selection of chemoagent for TACE. There is a lack of high-quality evidence on the selection of chemolipiodolization agent to date. As no significant differences in background characteristics were found between the epirubicin and miriplatin TACE groups, we consider our analyses to be useful. Although investigations in numerous patients in various stages of diseases are required in the future, it is believed that double-platinum therapy with DDP-H and miriplatin for BCLC-B HCC may be a useful treatment strategy.

\section{References}

1. Bruix J and Sherman M; American Association for the Study of Liver Diseases: Management of hepatocellular carcinoma: An update. Hepatology 53: 1020-1022, 2011.

2. Bruix J and Sherman M; Practice Guidelines Committee, American Association for the Study of Liver Diseases: Management of hepatocellular carcinoma. Hepatology 42: 1208-1236, 2005. 
3. Tommasini M, Colombo M, Sangiovanni A, Orefice S, Bignami P, Doci R and Gennari L: Intrahepatic doxorubicin in unresectable hepatocellular carcinoma. The unfavorable role of cirrhosis. Am J Clin Oncol 9: 8-11, 1986.

4. Ichida T, Kato M, Hayakawa A, Watanabe M, Igarashi K, Hata K, Doya Y, Miura M, Sato H and Asakura H: Treatment of hepatocellular carcinoma with a CDDP-epirubicin-lipiodol suspension: A pilot clinico-pharmacological study. Cancer Chemother Pharmacol 31 (Suppl): S51-S54, 1992

5. Aoyama K, Tsukishiro T, Okada K, Tsuchida T, Aiba N, Nambu S, Miyabayashi C, Yasuyama T, Higuchi K and Watanabe A: Evaluation of transcatheter arterial embolization with epirubicin-lipiodol emulsion for hepatocellular carcinoma. Cancer Chemother Pharmacol 31 (Suppl): S55-S59, 1992.

6. Colleoni M, Gaion F, Liessi G, Mastropasqua G and Nelli P. Arterial chemoembolization with epirubicin in unresectable hepatocellular carcinoma in cirrhosis. Oncol Rep 1: 1171-1175, 1994.

7. Ohnishi K, Tsuchiya S, Nakayama T, Hiyama Y, Iwama S, Goto N, Takashi M, Ohtsuki T, Kono K, Nakajima Y, et al: Arterial chemoembolization of hepatocellular carcinoma with mitomycin C microcapsules. Radiology 152: 51-55, 1984

8. Sasaki Y, Imaoka S, Kasugai H, Fujita M, Kawamoto S, Ishiguro S, Kojima J, Ishikawa O, Ohigashi H, Furukawa $\mathrm{H}$, et al: A new approach to chemoembolization therapy for hepatoma using ethiodized oil, cisplatin, and gelatin sponge. Cancer 60: 1194-1203, 1987.

9. Ishikawa T, Higuchi K, Kubota T, Seki K, Honma T, Yoshida T and Kamimura T: Prevention of intrahepatic distant recurrence by transcatheter arterial infusion chemotherapy with platinum agents for stage I/II hepatocellular carcinoma. Cancer 117: 4018-4025, 2011.

10. Ishikawa T, Kubota T, Abe S, Watanabe Y, Sugano T, Inoue R, Iwanaga A, Seki K, Honma T and Yoshida T: Hepatic arterial infusion chemotherapy with cisplatin before radical local treatment of early hepatocellular carcinoma (JIS score 0/1) improves survival. Ann Oncol 25: 1379-1384, 2014.

11. Yamada R, Sato M, Kawabata M, Nakatsuka H, Nakamura K and Takashima S: Hepatic artery embolization in 120 patients with unresectable hepatoma. Radiology 148: 397-401, 1983.

12. Matsui O, Kadoya M, Yoshikawa J, Gabata T, Arai K, Demachi H, Miyayama S, Takashima T, Unoura M and Kogayashi K: Small hepatocellular carcinoma: Treatment with subsegmental transcatheter arterial embolization. Radiology 188: 79-83, 1993.

13. Kim HC, Lee JH, Chung JW, Kang B, Yoon JH, Kim YJ, Lee HS, Jae HJ and Park JH: Transarterial chemoembolization with additional cisplatin infusion for hepatocellular carcinoma invading the hepatic vein. J Vasc Interv Radiol 24: 274-283, 2013.

14. Tanaka M, Yoshida S and Kimura K: Mechanism of inhibition of DNA polymerases by 4'-epiadriamycin and 4'-O-tetrahydropyranyladriamycin. Gan 74: 829-836, 1983.

15. Nagasue N, Yukaya H, Okamura J, Kuroda C, Kubo Y, Hirai K, Tanikawa K, Okita K, Ando K and Tamura K: Intra-arteria administration of epirubicin in the treatment of non-resectable hepatocellular carcinoma. Epirubicin study group for hepatocellular carcinoma. Cancer \& chemotherapy 13: 2786-2792, 1986 (In Japanese).
16. Yoshikawa M, Saisho H, Ebara M, Iijima T, Iwama S, Endo F, Kimura M, Shimamura Y, Suzuki Y, Nakano T, et al: A randomized trial of intrahepatic arterial infusion of 4'-epidoxorubicin with Lipiodol versus 4'-epidoxorubicin alone in the treatment of hepatocellular carcinoma. Cancer Chemother Pharmacol 33 (Suppl): S149-S152, 1994.

17. Maeda M, Uchida NA and Sasaki T: Liposoluble platinum (II) complexes with antitumor activity. Jpn J Cancer Res 77: 523-525, 1986.

18. Kishimoto S, Ohtani A, Fukuda H, Fukushima S and Takeuchi Y: Relation between intracellular accumulation and cytotoxic activity of cis- $[((1 \mathrm{R}, 2 \mathrm{R})-1,2$-cyclohexanediamine-N, N') bis(myristato)]platinum(II) suspended in Lipiodol. Biol Pharm Bull 26: 683-686, 2003.

19. Hanada M, Baba A, Tsutsumishita Y, Noguchi T, Yamaoka T, Chiba $\mathrm{N}$ and Nishikaku F: Intra-hepatic arterial administration with miriplatin suspended in an oily lymphographic agent inhibits the growth of tumors implanted in rat livers by inducing platinum-DNA adducts to form and massive apoptosis. Cancer Chemother Pharmacol 64: 473-483, 2009.

20. Hanada M, Baba A, Tsutsumishita Y, Noguchi T and Yamaoka T: Intra-hepatic arterial administration with miriplatin suspended in an oily lymphographic agent inhibits the growth of human hepatoma cells orthotopically implanted in nude rats. Cancer Sci 100: 189-194, 2009.

21. Okusaka T, Okada S, Nakanishi T, Fujiyama S and Kubo Y: Phase II trial of intra-arterial chemotherapy using a novel lipophilic platinum derivative (SM-11355) in patients with hepatocellular carcinoma. Invest New Drugs 22: 169-176, 2004.

22. Miyayama S, Yamashiro M, Shibata Y, Hashimoto M, Yoshida M, Tsuji K, Toshima F and Matsui O: Comparison of local control effects of superselective transcatheter arterial chemoembolization using epirubicin plus mitomycin $\mathrm{C}$ and miriplatin for hepatocellular carcinoma. Jpn J Radiol 30: 263-270, 2012.

23. Handa T, Imai Y, Sugawara K, Chikayama T, Nakazawa M, Ando S, Hamaoka K, Inao M, Nakayama N and Mochida S: Transcatheter arterial chemoembolization for hepatocellular carcinoma: Comparison of the therapeutic efficacies between miriplatin and epirubicin. Hepatol Res 44: 1072-1080, 2014.

24. Kamimura K, Suda T, Tamura Y, Takamura M, Yokoo T, Igarashi M, Kawai H, Yamagiwa S, Nomoto M and Aoyagi Y: Phase I study of miriplatin combined with transarterial chemotherapy using CDDP powder in patients with hepatocellular carcinoma. BMC Gastroenterol 12: 127, 2012. 